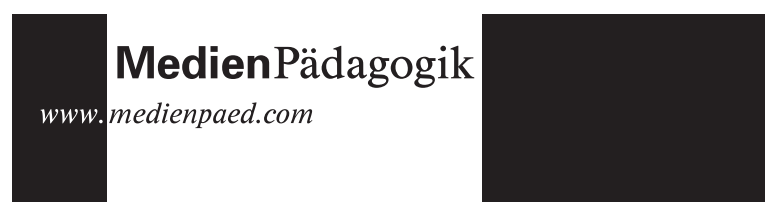

Hermann Astleitner, Iwan Pasuchin und Christian Wiesner

\section{Multimedia und Motivation - Modelle der Motivationspsychologie als Grundlage für die didaktische Mediengestaltung}

Aus der bisherigen Forschung zum Lernen mit Multimedia gibt es häufig widersprüchliche Ergebnisse, da zum Teil unerwartete Effekte aufgetreten sind oder wichtige lernrelevante Parameter ausser Acht gelassen wurden. Mängel lassen sich vor allem im Bereich der Motivation feststellen. Traditionelle Theorien zum Lernen mit Multimedia sind meist auf kognitive Faktoren aufgebaut und ignorieren, dass die Motivation eines Lernenden Lernressourcen signifikant beeinflussen kann. In dieser Arbeit werden theoretische Modelle kritisch besprochen. Die daraus gewonnenen Erkenntnisse münden in ein integratives theoretisches Modell, das motivationale Faktoren beim Lernen mit Multimedia extrahiert. Dieses Modell sollte zukünftige Forschung anregen, da es eine Erweiterung der populären kognitiven Theorie des Lernens mit Multimedia von Mayer (2001) darstellt. Das geschaffene Modell integriert zwei Typen von Variablen, die mentales Ressourcenmanagement (Aufmerksamkeit, Engagement, Monitoring) und motivationale Prozesse (Zielsetzung und Handlungskontrolle) betreffen. Es kann Forschern, Medienpädagogen, Unterrichts- und/oder Mediengestaltern einen Überblick über die Hauptfaktoren geben, die berücksichtigt werden müssen, wenn man multimediale Lernumgebungen motivational wirksam entwickeln will.

\section{Einleitung}

Eine der schwierigsten Aufgaben bei der Etablierung von multimedialen Lernumgebungen stellt das Motivieren von unmotivierten Lernenden dar. Diese Aufgabe wird umso schwieriger als die Forschung im Bereich Multimedia bisher motivationale Faktoren kaum berücksichtigt hat. Eine grundlegende Tatsache dieser Forschung im Bereich multimedialen Lernens ist, dass Studien, die ähnliche oder völlig gleiche Instruktionselemente testen, positive Effekte zeigen, andere jedoch keine oder sogar negative Effekte (vgl. beispielsweise die Metaanalyse von Liao, 1999). Dieser Umstand ist bedingt durch die vielen sich gegenseitig beeinflussenden Faktoren (multimedialer Input, kognitive Bearbeitung, Lernerdynamik, etc.), deren Beziehungen theoretisch unterspezifiziert sind. Um die Wirksamkeit von multimedialem Lernen bestimmen zu können, muss eine Theorie generiert werden, die so viele relevante Faktoren wie möglich für das multimediale Lernen berücksichtigt, im Besonderen aber auch motivationale Faktoren.

Im Moment befassen sich einige Ansätze mit motivationalen Faktoren beim multimedialen Lernen. Diese vorhandenen Theorien müssen in Bezug auf ihre Kapazität, Theorie, Forschung und Praxis anzuregen, bewertet werden. Diese Ansätze sind in der Forschung im Bereich multimediales Lernen meist noch unbekannt und stammen von Malone/Lepper (1987), aus einer Kombination eines Modells von Motivation bei selbst reguliertem Lernen und dem ARCS-Ansatz (Keller, 1999; Rheinberg/Vollmeyer/Rollett, 2000), aus einem Modell der integrierten multimedialen Effekte von Hede (2002) und schliesslich aus Mayers (2001) kognitiver Theorie des multimedialen Lernens, welche, als zentrales Ergebnis der vorliegenden Arbeit, um motivationale Faktoren zu erweitern ist.

Die Methode dieses Beitrages besteht aus der Bewertung theoretischer Ansätze und auf der Schaffung eines alternativen theoretischen Rahmens. Dabei sind zunächst die üblichen Kriterien sozialwissenschaftlicher Forschung zu berücksichtigen, wie logische Konsistenz, Informationsgehalt, Sparsamkeit in der Parameterverwendung, etc. Die zu analysierenden Ansätze werden deskriptiv mit diesen Kriterien verglichen. Werden Diskrepanzen zwischen den Kriterien und den Merkmalen der Ansätze entdeckt, dann wird Kritik geäussert.

Beim Finden eines eigenen theoretischen Ansatzes wird die geäusserte Kritik genutzt, um Schwachstellen und/oder Anknüpfungspunkte zu identifizieren. Das so gewonnene Modell wird hinsichtlich der Exklusivität und 
erschöpfenden Darstellung des Sachverhaltes überprüft und gegebenenfalls kalibriert. Dabei werden gängige Verfahren der qualitativen Sozialforschung genutzt und Querverbindungen zur quantitativ-empirischen Forschung hergestellt (vgl. Miles/Huberman, 1994)

\section{Der Instructional Design Ansatz von Malone/Lepper (1987)}

Komponenten. Malone/Lepper (1987) identifizierten vier Faktoren (Herausforderung, Neugier, Kontrolle und Fantasie), mit welchen eine multimediale Lernumgebung motivierend gestaltet werden kann. Um die Lernenden weder zu langweilen noch zu frustrieren und um herausfordernd zu sein, sollten Aktivitäten kontinuierlich auf einem optimalen Schwierigkeitsgrad ablaufen. Um Neugier zu wecken, können audiovisuelle technische Hilfsmittel genutzt werden, sowie die Präsentation von unvollständiger bzw. widersprüchlicher Information bezogen auf die kognitive Struktur der Lernenden. Die Kontrolle auf Seiten der Lernenden kann dadurch gefördert werden, dass die Lernergebnisse vom eigenen Handeln des Lernenden mitbestimmt werden. Schliesslich können die Lernenden durch fantasievolle und virtuelle Aktivitäten und Kontexte intrinsisch motiviert werden.

Bewertung. Der Instruktionsansatz von Malone/Lepper (1987) basiert zu einem gewissen Grad auf traditionellen Motivationstheorien und ist für Instruktions- oder Mediendesigner hauptsächlich als Anleitung bzw. Orientierungshilfe zu verstehen. Diese Anleitungen werden auf multimediales Lernen bezogen und inkludieren z. B. audiovisuelle Strategien. Sie sind ausserdem umfassend, ausschliessend und repräsentieren motivational wirksame Parameter. Allerdings regte dieses Modell kaum empirische Forschung an. Obwohl dieser Ansatz keine Theorie darstellt, wie Elemente einer multimedialen Lernumgebung mit verschiedenen Variablen zusammenhängen, beschreibt der Instruktionsansatz mehr oder weniger gültige motivationale Prozesse bei einem Lernenden und ihre Effekte auf Lernen. Dies ist in diesem Modell durch die Berücksichtigung von neueren lerntheoretischen Entwicklungen erkennbar, wie beispielsweise dem selbst regulierten Lernen. Da selbst reguliertes Lernen mittlerweile ein bedeutsames lerntheoretisches Paradigma darstellt, bedarf die Selbstregulation von Motivation besonderer Aufmerksamkeit. Der Ansatz von Malone/ Lepper (1987) hat nur eine beschreibende Funktion: Er kategorisiert und fasst motivational relevante Faktoren des Lernens unter Berücksichtigung multimedialer Aspekte zusammen. Kritisch anzumerken ist, dass diese
Faktoren nicht innerhalb einer üblichen Lern- und/oder Motivationstheorie aufeinander bezogen werden. Nur solch eine Theorie, die unterschiedliche motivationsrelevante Grössen miteinander kombiniert, könnte eine erklärende und überzeugende Grundlage für die vielen möglichen Wechselwirkungseffekte der in diesem Modell identifizierten Faktoren bieten.

\section{Ein kombiniertes Modell: ARCS und selbst reguliertes Lernen}

Die Defizite aus dem Ansatz von Malone/Lepper (1987) können reduziert werden, indem ein aktuelles Motivationsmodell zum selbst regulierten Lernen von Rheinberg/Vollmeyer/Rollett (2000) mit dem A(ttention) $\mathrm{R}$ (elevance) C(onfidence) S(atisfaction)-Ansatz von Keller (1999) kombiniert wird.

Komponenten. Der ARCS-Ansatz postuliert eine Reihe von Motivationsbedingungen, die auch in multimedialen Lernumgebungen aktiviert werden können: Damit Motivation überhaupt erst wirksam werden kann, muss Aufmerksamkeit geweckt und aufrechterhalten werden. Aufmerksamkeit kann beispielsweise strategisch durch Förderung der Wissbegierde, durch überraschende und inkongruente Elemente oder durch abwechselnde Instruktion erzeugt werden. Relevanz wird durch das Verbinden der Bedürnnisse der Lernenden und den Lehrinhalten hergestellt. Relevanz kann durch das Abstimmen der Lehrstrategien auf verschiedene Motivationsprofile wie auch durch das Transparentmachen von Zielen und des Nutzens für den Lernenden erzeugt werden. Vertrauen wird durch eine positive Erfolgserwartung gefördert. Selbstvertrauen kann durch transparente Leistungsanforderungen, selbst gewählte Leistungsstandards sowie durch Rückmeldungen - wobei hier die Fähigkeit und Anstrengung der Lernenden als die Determinante von Erfolg deutlich gemacht werden soll aufgebaut werden. Zufriedenheit entsteht, wenn die Ergebnisse den geleisteten Anstrengungen entsprechen. Zufriedenheit kann dadurch gefördert werden, dass Lernenden die Möglichkeit gegeben wird, ihr neu erworbenes Wissen in reellen bzw. simulierten Situationen anzuwenden.

Das motivationale Modell selbst regulierten Lernens von Rheinberg, Vollmeyer und Rollet (2000) beschreibt folgenden sich wiederholenden Prozess: Ein selbst reguliert Lernender findet unterschiedliche Ziele erstrebenswert, dann - aufgrund von Information aus der multimedialen Lernumgebung - vergleicht er die unterschiedlichen Ziele in Hinblick auf Erwartungen und/oder Anreizwerten und wählt ein Ziel als Absicht für das Handeln aus. Nachdem eine Absicht gegeben ist, führt der Lernende 
Aktivitäten aus, um das Ziel zu erreichen, welches mit seiner Absicht verbunden ist. Um erfolgreich $\mathrm{zu}$ sein, begleiten Handlungskontrollprozesse die Umwandlung der Absicht in Handlungen. Während des Handelns erzeugen neue Erfahrungen einen neuen unbestimmten Zielstatus und so weiter. Der sich wiederholende Prozess der Motivation beim selbst regulierten Lernen wird eingestellt, wenn alle vorgegebenen Ziele erreicht sind und/oder wenn keine neuen Ziele erscheinen. Innerhalb dieses kombinierten Modells wird angenommen, dass auf einer ersten Stufe sich ein selbst reguliert Lernender in einem unbestimmten Zielstatus befindet. Dieser Status wird in eine handlungsleitende Absicht transformiert, wenn niedrige Situations-Ergebnis-Erwartung (SOE), hohe Handlungs-ErgebnisErwartung (AOE), hohe Ergebnis-Folge-Erwartung (OCE), und hoher Anreizwert (I) für ein bestimmtes Ziel gegeben sind. Auf einer zweiten Stufe wird die Absicht in konkrete Handlungen umgewandelt, wobei die Handlungskontrolle (Aufmerksamkeit, Enkodieren, Kognition, Emotion, Motivation und Umweltkontrollmechanismus) auf die Absicht einwirkt.

Zentrale Annahme des kombinierten Modells ist es jetzt, dass der selbst regulierte Lernende auf den unterschiedlichen Stufen mittels instruktionalen Strategien des ARCS-Ansatzes innerhalb der Lernumgebung unterstützt werden kann. Diese Strategien können multimediale Ausformungen annehmen. Instruktionale Strategien, die den Parameter Selbstvertrauen betreffen, können mit SOE (d. s. instruktionale Strategien, die günstige Attribuierungsmuster fördern) und mit AOE (d. s. instruktionale Strategien, die Erwartungen für Erfolg und eine Einstellung zur Herausforderung stimulieren) verbunden werden. Wenn beispielsweise Lernenden gesagt wird, dass Anstrengung notwendig ist, um beim Lernen erfolgreich zu sein, dann wird SOE niedrig gehalten, weil die Lernenden nicht glauben, dass eine vorgegebene Situation zu einem erwünschten Ergebnis ohne eigene Anstrengung führen wird. AOE steht für das Konzept der «Wahrscheinlichkeit von Erfolg», das durch das Mitteilen von Kriterien und das Bereitstellen vielfacher Leistungsstufen beeinflusst werden kann. OCE und Anreizwerte können mit Instruktionsstrategien stimuliert werden, welche den Parameter Zufriedenheit betreffen und handeln von Gleichheit (d. s. instruktionale Strategien, die eine konsistente Ergebnis-KonsequenzBeziehung ausdrücken) oder befassen sich mit natürlichen und positiven Konsequenzen (für die Erzeugung von Anreizwerten). Instruktionale Strategien, die den Parameter Aufmerksamkeit betreffen, können mit Handlungskontrolle in Verbindung gesetzt werden. Die Ziel-Orientierung als Teil der auf Relevanz bezogenen Instruktionsstrategien sollte eine Wirkung auf das Enkodieren und die kognitive Kontrolle haben, da es den Lernenden mit Information versorgt, was für das Lernen und Verstehen wichtig ist. Motiv-Anpassung stellt eine Instruktionsstrategie für die Unterstützung von vor allem Motivationskontrolle dar, weil die Lernenden hingewiesen werden, sich auf ihre persönlichen Bedürfnisse und ihre Wünsche zu konzentrieren. Schliesslich können Instruktionsstrategien, die den Parameter Selbstvertrauen ansprechen, mit einer Umgebungskontrolle in Verbindung gebracht werden, weil vertraute Umgebungen geringere kognitive Anstrengung (und Handlungskontrolle) für die Handlung benötigen als unbekannte Umgebungen.

Bewertung. Dieses kombinierte Modell kann einerseits auf Grund der zwei unterschiedlichen theoretischen Hintergründe kritisiert werden und andererseits weil es explizit keine Elemente aus multimedialen Lernumgebungen (beispielsweise audiovisuelle Bestandteile von Lernumgebungen) berücksichtigt. Das Modell des selbst regulierten Lernens basiert hinsichtlich der Motivation hauptsächlich auf kognitivistischen Theorien, während der ARCS-Ansatz der ersten Generation im Allgemeinen eher eine behavioristische Sichtweise von Motivation verfolgt, was Paradigmeninkompatibilitäten und theoretische Integrationsprobleme mit sich bringt. In diesem kombinierten Modell gibt es auch keine Aussagen oder Axiome, die ausführlich und begründet spezifische Instruktionsstrategien und damit kognitive Prozesse zur Erregung und Aufrechterhaltung von Motivation beschreiben. Die vorgegebenen Instruktionsstrategien des ARCS-Ansatzes beziehen sich mehr oder weniger schlüssig bzw. theoretisch nur eingeschränkt konsistent auf die Elemente eines motivationalen Prozesses, der vom Ansatz selbst regulierten Lernens postuliert wird. Dieses Problem könnte vom hohen Grad der Allgemeingültigkeit und damit vom Ziel des ARCS-Ansatzes abhängen. Die ARCSStrategien repräsentieren Instruktionsanleitungen, um motivationale Probleme in der pädagogischen Praxis zu lösen. Dazu benötigt es allgemeine Strategien, die leicht den pädagogischen Problemen angepasst werden können. Die stärkere Mikroorientierung des Ansatzes selbst regulierten Lernens leistet das nicht, was wieder zu theoretischen und methodischen Inkompatibilitäten führen kann. Ein weiteres Problem bei diesem kombinierten Modell entsteht durch fehlende didaktische Anleitung im multimedialen Anwendungsbereich. Innerhalb des ARCS-Ansatzes werden multimediale Aspekte (beispielsweise audiovisuelle Medieneffekte) zwar 
berücksichtigt, sie werden jedoch nur auf den Parameter Aufmerksamkeit bezogen. Allerdings können multimediale Gestaltungselemente auch den Grad der Realitätsnähe einer Lernumgebung verbessern (zum Beispiel durch Darstellung von wirklichen Lebenssituationen oder realistischen Animationen). Auch kann die Realitätsnähe andere Parameter anregen, wie beispielsweise die Vertrautheit des Lernkontextes, was wiederum mit Selbstvertrauen verbunden ist. Da solche Einflüsse nicht im vorgestellten kombinierten Modell postuliert werden, verursacht dies insbesondere theoretische Probleme in Bezug auf eine umfassende und integrative Betrachtung und Erklärung multimedialen Lernens.

\section{Ein integriertes Modell multimedialen Lernens}

Komponenten. Das kombinierte Modell von Hede (2002) besteht aus mehreren Faktorengruppen. Die erste Gruppe ist mit dem Input des Lernprozesses verknüpft (Lehrmaterial). Multimedialer Input erfolgt - nach Annahmen dieses Modells - visuell und auditiv (visueller Input meint Texte, Bilder, Diagramme, Video und Animation und auditiver Input besteht aus Erzählungen oder Kommentaren, Anweisungen oder Musik). Multimediale Lernumgebungen stellen dem Lernenden verschiedene Ausprägungen der Lernerkontrolle über diese Inputs zur Verfügung beispielsweise durch bestimmte Designmerkmale und Links. Dem Lernenden stehen auch verschiedene Stufen von Interaktivität zur Verfügung, welche entweder vom ihm selbst oder durch das Programm gesteuert werden können.

Die zweite Gruppe von Faktoren bezieht sich auf die Bearbeitung der Information und basiert auf der Aufmerksamkeit sowie dem Arbeitsgedächtnis. Aufmerksamkeit dient dazu, um die Konzentration des Lernenden auf den Input zu richten. Informationsverarbeitung findet auf unterschiedliche Weise im Arbeitsgedächtnis statt. Grundsätzlich ermöglicht eine als dual angenommene Kodierung die gleichzeitige Bearbeitung auditiver und visueller Inputs. Ausserdem muss die kognitive Belastung berücksichtigt werden: Kognitive Überlastung entsteht, wenn der Input die beschränkte Kapazität des Arbeitsgedächtnisses überschreitet. Einen weiteren Parameter stellt eine kognitiv-wahrnehmungsbezogene Störung (z. B. durch multimediale «Geräusche», Hintergrundmusik, etc.) dar, wenn beispielsweise eine Quelle die semantische Informationsbearbeitung einer anderen Quelle behindert. Das Behalten von Information hängt auch von der Wiederholung des Gelernten ab. Schliesslich repräsentiert das Arbeits- gedächtnis eine kognitive Schaltzentrale, wo verbale und visuelle Repräsentationen miteinander verbunden werden. Bezüglich der Lernerdynamik gibt es die intrinsisch und extrinsisch motivierenden Bestandteile, die das kognitive Engagement beeinflussen - also den Prozess, bei dem die Lernenden ihre Lerntätigkeiten, Lernzeit sowie ihre Anstrengung bestimmen. Auch der Lernstil der Lernenden muss berücksichtigt werden, d. h. wie unterschiedliche (z. B. feldabhängige oder feldunabhängige, oberflächlich oder tiefenstrukturell verarbeitende und aktive oder passive) Lernende auf Multimedia reagieren.

Die letzte Gruppe von Faktoren besteht aus vier Elementen: Intelligenz, Reflexion, Langzeitgedächtnis und Lernen. Von Intelligenz wird angenommen, dass viele unterschiedliche Formen und Ausprägungen existieren. Eine Multimedia-Präsentation sollte daher möglichst mehrere Formen der Intelligenz anregen. Reflexion meint die Bewertung des eigenen Denkens und Lernens. Das Langzeitgedächtnis erhält Information aus dem Arbeitsgedächtnis und unterstützt dieses wiederum durch bereits gespeichertes Wissen beim Lernen. Im Langzeitgedächtnis wird zwischen deklarativem, konzeptuellem und prozeduralem Wissen unterschieden. Schliesslich umfasst Lernen das Verstehen des multimedial aufbereitenden Materials sowie die Fähigkeit, das erlernte Wissen abzurufen und anzuwenden.

Bewertung. Das integrierte Modell von Hede (2002) berücksichtigt umfassend multimediale Aspekte sowie auch motivationale Faktoren. Allerdings kann dieses Modell als eklektizistisch bezeichnet werden. Ausserdem ist es durch seine Vielzahl von Bestandteilen und Beziehungen zwischen diesen Bestandteilen als nicht sparsam zu bezeichnen. Das Modell ist eklektizistisch, weil es einerseits Variablen aus unterschiedlichen theoretischen Hintergründen integriert ohne diese plausibel (auf der Basis eines eigenen theoretischen Konzeptes) zu verbinden und andererseits stellt es kein innovatives Konzept dar, welches neue Perspektiven in die Multimediaforschung bringt. Dieses Modell kann wenig dazu beitragen, dass die vielen verschiedenen Faktoren $\mathrm{zu}$ einer akzeptablen und damit erforschbaren Zahl reduziert werden bzw. dass konfundierte Effekte verhindert werden, die innerhalb experimenteller Multimediaforschung immer noch vorherrschend sind.

Die motivationale Erweiterung von Mayer's (2001) kognitivem Modell Komponenten. Die kritischen Anmerkungen zum Modell von Hede (2002) wären $\mathrm{zu}$ vermeiden, wenn eine wohlbegründete und gut fundierte multi- 
mediale Lerntheorie berücksichtigt und diese mit nur wenigen Parametern erweitert wird. Solch eine theoretische Basis wird von der kognitiven Theorie des Lernens mit Multimedia von Mayer (2001) geleistet. Diese Theorie basiert auf den folgenden Annahmen: (a) das Arbeitsgedächtnis umfasst voneinander unabhängige auditive und visuelle Kanäle; (b) jeder Kanal im Arbeitsgedächtnis hat eine beschränkte Kapazität (konsistent mit der cognitive load-Theorie); (c) Menschen haben getrennte Systeme für die Darstellung der verbalen und nichtverbalen Information (konsistent mit der Theorie der dualen Kodierung); und (d) bedeutungsvolles Lernen geschieht dann, wenn ein Lernender in jedem Kanal relevante Information auswählt, die Information in den Kanälen in einer zusammenhängenden Repräsentation organisiert und Verbindungen zwischen verschiedenen Repräsentationen herstellt. Diese Theorie führte durch eine Vielzahl von Forschungsstudien zu unterschiedlichen, relativ gut bewiesenen Prinzipien des Lernens mit Multimedia (vgl. z. B. Plass/Chun/Mayer/Leutner, 1998). Die wichtigsten von dieser Theorie abgeleiteten und international beachteten Prinzipien der Gestaltung von multimedialen Lernumgebungen sind: (a) Prinzip der geteilten Aufmerksamkeit (Lernende lernen besser, wenn sie ihre Aufmerksamkeit nicht zwischen vielfachen Quellen von Information teilen müssen); (b) Modalitätsprinzip (Lernende lernen besser, wenn verbale Auskunft eher auditiv dargestellt wird als visuell als sichtbarer Text); (c) Prinzip der räumlichen Nähe (Lernende lernen besser, wenn sichtbarer Text und visuelle Materialien nicht getrennt sondern miteinander dargeboten werden); oder (d) Prinzip der zeitlichen Nähe (Lernende lernen besser, wenn verbale und visuelle Materialien zeitlich synchronisiert werden und nicht zeitlich getrennt dargeboten werden).

Bewertung. Das Hauptproblem bei Mayer's Ansatz (2001) liegt in der fehlenden Berücksichtigung motivationaler Aspekte beim Lernen mit Multimedia. In multimedialen Lernumgebungen können einige als kognitiv wirksam angenommene Elemente auch nicht-kognitiver Art sein. Beispielsweise hat Video einen grösseren motivationalen Wert als Audio-Information, da es dynamische Bilder, Farben, etc. aufweist. Auch kann Audiooder Videoinformation als Lernhilfe und -unterstützung motivationale Qualität besitzen, um beispielsweise die Angst vor Misserfolg zu reduzieren. Harp/Mayer (1997) haben in weiteren Arbeiten zwischen «kognitivem Interesse» (basiert auf struktureller Kohärenz von Information) und «emotionalem Interesse» (basiert auf Aufmerksamkeit und Neugier) unterschieden, die durch unterschiedliche multimediale Elemente angeregt werden. Ausserdem befassen sich Ansätze von z. B. Astleitner/ Leutner (2000) mit motivationalen Aspekten von multimedialen Instruktionselementen. Diese motivationalen Elemente sind wichtig, weil (a) Motivation Lernen bedeutsam beeinflusst; (b) motivationale Prozesse vermutlich Ressourcen aus dem Gedächtnis benötigen und deshalb die kognitive Belastung verringern oder erhöhen; und (c) es eine direkte Verbindung zwischen kognitiven und motivationalen Variablen gibt, nämlich Aufmerksamkeit. Aufmerksamkeit stellt ein besonders wichtiges Element dar und das sowohl für kognitive als auch für motivationale Lernmodelle. Eine Verringerung der Mängeln des Ansatzes von Mayer (2001) könnte durch eine integrative multimediale Lerntheorie erreicht werden, welche kognitive und motivationale Aspekte der Speicherauslastung im Arbeitsgedächtnis sowie der Einspeicherung und des Abrufes aus dem Langzeitgedächtnis in Verbindung mit Lernen bringt.

\section{Belege für motivationale Parameter im Arbeitsgedächtnis}

Grundsätzlich muss zunächst angenommen werden, dass nicht nur Wörter, Bilder etc. sowie ihre Organisation als mentale Modelle und ihre Integration in das individuelle Wissen dem Arbeitsgedächtnis Kapazität abverlangen, sondern das gilt, dass auch andere Ressourcen, welche Lernprozesse regeln und beeinflussen, sich direkt auf die Kapazität des Arbeitsgedächtnisses auswirken. Solche Ressourcen werden Kosten- und Nutzenanalysen, Willens- bzw. Handlungskontrollprozessen und nicht-kognitiven, emotionalen Elementen im Gedächtnis zugeordnet.

Menschliches Lernen kann durch innerliche und äusserliche Beschränkung als Prozess einer Ressourcenverwaltung angesehen werden, die von motivationalen Parametern gesteuert wird (beispielsweise durch Erwartungen und Anreizwerte). Das Ziel dieses Prozesses besteht primär darin, die Verwendung von vorhandenen Ressourcen zu optimieren. Handlungen sind optimal oder maximal effizient, wenn der Ressourceneffekt möglichst klein und der Ressourcenzuwachs möglichst gross ist. Notwendige Verbesserungen eines Lernprozesses können Handlungen erfordern, welche zusätzlich Ressourcen verbrauchen. Auch das Überwachen (Monitoring) einer Aufgabenbearbeitung benötigt kognitive Ressourcen. Einzig Automatisierung reduziert diesen Ressourcenverbrauch, so dass kognitive Kapazität für das Lernen frei wird.

Automatische Informationsverarbeitung steht beim Lernen oft in einem Naheverhältnis zu motivationalen Parametern, hier besonders $\mathrm{zu}$ hand- 
lungssteuernden Facetten der Motivation. Kuhl (1985) postulierte, dass nicht nur Wörter, Bilder und Strukturen im Arbeitsgedächtnis dargestellt werden, sondern auch Absichten, Wünsche, Werte, etc. Willenskraft verhindert, dass andere Absichten dominant werden, bevor ein gegenwärtiges Ziel erreicht ist. Entsprechend dem Modell der Handlungskontrolle von Kuhl (1985) basieren willentliche Prozesse auf folgenden Komponenten: (a) aktive, selektive Aufmerksamkeit: sie fördert eine Informationsbearbeitung, die gerade die gegenwärtige Absicht unterstützt und die Informationsbearbeitung konkurrierender Absichten hemmt; (b) Enkodierungskontrolle: sie erhöht die Wirkung der Willenskraft, indem selektiv jene Merkmale von einem Reiz kodiert werden, die in Zusammenhang mit der gegenwärtigen Absicht stehen; (c) Emotionskontrolle: sie hemmt emotionale Zustände, die die Leistungsfähigkeit der Willenskraft beeinträchtigen könnten; (d) Motivationskontrolle: sie betrifft Feedback von selbstregulativen Prozessen zur eigenen motivationalen Befindlichkeit; (e) Kontrolle der Umgebung: sie nutzt Emotionskontrolle und Motivationskontrolle zur Manipulation der Umgebung; und (f) Sparsamkeit von Informationsverarbeitung: sie betrifft einen Aspekt von willentlicher Kontrolle, die sich auf Regeln der Informationsbearbeitung bezieht.

Bower (1987) postulierte, dass Gedächtnissysteme auch Knoten enthalten, die emotional und nicht-kognitiv sind. Sobald diese aktiviert sind, beeinflussen solche Emotionsknoten den weiteren Weg der Informationsbearbeitung, was zu einer einseitigen kognitiven Entscheidungsfindung führen kann. Eine einseitige Entscheidungsfindung im Gedächtnis kann dem Bewusstsein zugänglich und unter willentlicher Kontrolle sein, sowie die Ressourcenverteilung der Aufmerksamkeit beeinflussen. Wenn sie aber automatisiert ist, dann ist diese Entscheidungsfindung unbewusst, unbeabsichtigt und unabhängig von der Verteilung der Ressourcen beim Enkodieren. An dieser Stelle soll jedoch erwähnt werden, dass es ein Kontinuum zwischen Automatisierung und Kontrolle gibt, und dass es sich dabei um keine starre Dichotomie handelt. Einseitige Entscheidungsfindung kann als gegeben angenommen werden, wenn (a) Fachwissen nicht vorhanden war; (b) Fachwissen zwar vorhanden oder falsch angewandt wurde; (c) negative, auf sich selbst bezogene Gedanken im Arbeitsgedächtnis gegeben sind; oder (d) die Informationsverarbeitung störende Prozesse gegeben sind, so z. B. Gedanken, die irrelevant für das Problemlösen sind.

\section{Das erweiterte Modell motivationalen multimedialen Lernens}

Kosten- und Nutzenanalysen, Willens- bzw. Handlungskontrollprozesse und nicht-kognitive Elemente sind Teil der Vorgänge im Arbeitsgedächtnis und sollten deswegen in einem erweiternten Lernmodell mit Multimedia berücksichtigt werden. Um ein integriertes und umfassendes Modell über das Lernen mit Multimedia und Motivation $\mathrm{zu}$ finden, wurde der theoretische Ansatz von Mayer (2001, S. 44), der das Hauptgerüst darstellt, mit Ansätzen von Keller (1999), Hoogeveen (1997) und Hede (2002) verbunden, wobei eigene theoretische Grössen geschaffen wurden. Abbildung 1 zeigt das geschaffene Modell zur Integration von Motivation beim multimedialen Lernen. Darin wird angenommen, dass mentales Ressourcenmanagement und motivationale Prozesse die von Mayer (2001) postulierten mentalen Aktivitäten (Auswahl, Organisation und Integration) und mentale Modelle (verbaler und bildlicher Prägung) beeinflussen. Mentale Aktivitäten hängen vom mentalen Ressourcenmanagement ab, welches wiederum selbst von motivationalen Prozessen abhängt. Mentales Ressourcenmanagement ist eng mit Aufmerksamkeit, Engagement und Monitoring verknüpft. Motivationale Prozesse bestehen aus Zielsetzung und Handlungskontrolle.

Mentales Ressourcen-Management. Aufmerksamkeit beeinflusst die Kapazität des Arbeitsgedächtnisses, die einer bestimmten Aufgabe innerhalb einer vorgegebenen Zeit gewidmet wird. Engagement betrifft die Anzahl mentaler Aktivitäten in Hinblick auf eine bestimme Aufgabe innerhalb einer bestimmten Zeit. Monitoring hat die Funktion, die Aufmerksamkeit und das Engagement für eine bestimmte Aufgabe in einer vorgegebenen Zeit mittels Bewertung des Erfolges der mentalen Aktivitäten zu steuern. Zielsetzung betrifft das Überdenken eigener Erwartungen und Anreizwerte in Hinblick auf eine Aufgabe. Die Aufgabe, für die der grösste Wert der Kombination aus Erwartung und Wert resultiert, wird als auszuführende Absicht ausgewählt. Handlungskontrolle kann die aktuell gewählte Absicht - für das Erreichen eines bestimmten Zieles mittels einer entsprechenden Aufgabe - vor möglichen weiteren Alternativen abschirmen. Basierend auf diesen Bestandteilen des erweiterten Modells, können folgende Beziehungen zwischen mentalen Aktivitäten, mentalem Ressourcenmanagement und motivationalen Prozessen angenommen werden: Die verfügbare Kapazität des Arbeitsgedächtnisses, die Anzahl der Aktivitäten im Arbeitsgedächtnis und die Qualität der im Arbeitsgedächtnis statt- 
findenden Tätigkeiten stehen in enger Beziehung mit einer erfolgreichen mentalen Aktivität.

Wenn keine Aufmerksamkeit auf eine Aufgabe gerichtet ist, dann kann keine Informationsverarbeitung bezüglich dieser Aufgabe stattfinden und die Aufmerksamkeit verschiebt sich von relevanten zu aufgabenirrelevanten Aspekten innerhalb einer Lernsituation. In diesem Fall wird die Informationsverarbeitung gestört bzw. unterbrochen und vorläufige Lernerfolge und deren Ergebnisse können teilweise oder vollkommen vergessen werden. Dies führt zur ersten Annahme, dass aufgrund fehlender Aufmerksamkeit mentale Aktivitäten nicht abgeschlossen werden können (Wirkung auf Vollendung).

Wenn Aufmerksamkeit auf eine Aufgabe gerichtet ist, aber das Engagement nicht oder nur gering ausgeprägt ist, dann wird die Kapazität des Arbeitsgedächtnisses nicht zur Genüge für die Informationsverarbeitung genutzt. Im Arbeitsgedächtnis geschieht dann relativ wenig, damit werden keine oder nur wenige mentale Aktivitäten durchgeführt, keine oder nur wenige Begriffe oder Regeln werden erstellt oder erworben und das Wissen wird nicht ausreichend strukturiert bzw. nicht genügend mit bereits vorhandenem Wissen verknüpft, etc. Die daraus resultierende zweite Annahme lautet daher, dass es aufgrund fehlenden Engagements mehr Zeit bedarf, mentale Aktivitäten auszuführen (Wirkung auf Zeit).

Wenn Aufmerksamkeit und Engagement vorhanden sind, aber keine oder nur geringes Monitoring (Überwachung) gegeben ist, dann ist die Leistungsfähigkeit des Arbeitsgedächtnisses niedrig. Monitoring soll sicherstellen, dass der Erfolg der mentalen Aktivitäten in Form einer kontinuierlichen Selbstbewertung hergestellt wird. Auf diese Weise soll Aufmerksamkeit und Engagement so gesteuert werden, dass die Tätigkeiten im Arbeitsgedächtnis optimal die vorgegebene Absicht unterstützen. Dies führt zur dritten Annahme, dass durch das fehlende Monitoring eine mentale Aktivität ohne ausreichende Effektivität durchgeführt wird (Wirkung auf Erfolg).

Motivationale Prozesse. Zielsetzung hat die Aufgabe, mentales Ressourcenmanagement in Hinblick auf eine bestimmte Aufgabe, die mit dem jeweiligen Ziel in Verbindung steht, zu regulieren. Wenn die Überprüfung einer bestimmten Aufgabe durch individuelle Erwartungen und Anreizwerte die Absicht herbeiführt, an jener Aufgabe zu arbeiten, dann werden Aufmerksamkeit, Engagement und Monitoring zur Bearbeitung jener Aufgabe aktiviert. Zielsetzung findet während des Lernens dauerhaft statt und erhält auch aus den Ergebnissen des Monitoring ein Feedback, ob und wie gut Ziele erreicht werden. Handlungskontrolle hat die Funktion, mentales Ressourcenmanagement für eine bestimmte Aufgabe über einen bestimmten Zeitraum aktiv zu halten. Handlungskontrolle variiert personspezifisch, so können handlungsorientierte und lageorientierte Personen unterschieden werden. Handlungsorientierte Personen führen einen grösseren Anteil ihrer Absichten aus oder erleichtern lernrelevante Entscheidungsfindungen, indem sie aktiv ihre eigene Motivation für die vorläufig ausgewählte Alternative steigern.

Abbildung 1. Ein Modell von Multimedia, Lernen und Motivation als Erweiterung des Ansatzes von Mayer (2001, S. 44)

\begin{tabular}{|l|l|}
\hline $\begin{array}{l}\text { Multimedia } \\
\text { Präsentation } \\
\text { Worte } \\
\ldots . .\end{array}$ & $\begin{array}{l}\text { Sensorisches } \\
\text { Gedächtnis } \\
\text { Ohren } \\
.\end{array}$ \\
\hline
\end{tabular}

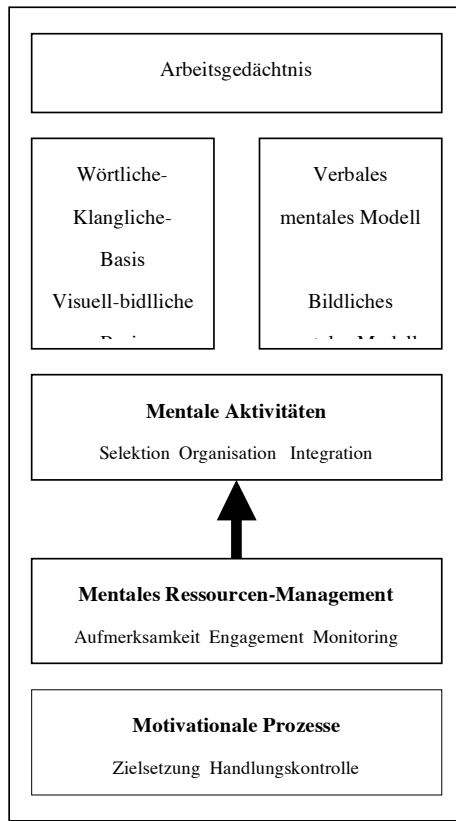

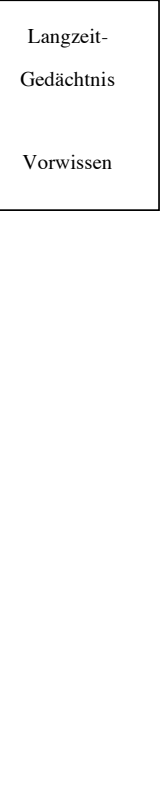




\section{Vorhersagen, die vom Modell abgeleitet werden können}

Allgemeine Voraussagen. Das dargestellte erweiterte Modell kann unterschiedliche Forschungsfragen im Bereich Multimedia anregen.

In einem ersten Schritt sollten jene Forschungen, die sich mit motivationaler Qualität von Multimedia-Elementen befassen, zu diesem Modell in Beziehung gesetzt werden, um die Konstruktvalidität und empirische Validität der Bestandteile des Modells bewerten zu können. Beispielsweise wären empirische Studien zu motivationalen Merkmalen in MultimediaLernumgebungen von Malone/Lepper (1987) oder Song/Keller (2001) zu integrieren. Diese können mit dem dargestellten erweiterten Modell verknüpft und Elemente einer multimedialen Lernumgebung können somit auf die postulierten theoretischen Beziehungen bezogen werden. Solche motivierenden Merkmale können zum Beispiel Aussagen der Nützlichkeit des Inhalts (bezogen auf die Zielsetzung) bzw. auditive oder visuelle Effekte (bezogen auf die Aufmerksamkeit) enthalten.

Zweitens könnte das erweiterte Modell zur ATI(aptitude-treatment-interaction)-Forschung anregen, indem man Persönlichkeitsmerkmale berücksichtigt und mit den dargestellten theoretischen Bestandteilen verbindet. Zielsetzung hängt beispielsweise davon ab, ob ein Lernender erfolgs- oder misserfolgsorientiert ist. Denn erfolgsorientierte Lernende konzentrieren sich im Vergleich zu misserfolgsorientierten Lernenden mehr auf realistische und erreichbare Ziele. Handlungskontrolle unterscheidet sich je nachdem, ob ein Lernender handlungsorientiert oder lageorientiert ist. Handlungsorientierte Lernende benutzen selektive Aufmerksamkeit, um jene Teile einer Lernumgebung zu nutzen, die eine aktuelle Absicht unterstützen, während lageorientierte Lernende sich auf andere Teile einer Lernumgebung konzentrieren (Kuhl, 1985).

Drittens sollten sich empirische Studien besonders auf die Frage konzentrieren, ob durch die Berücksichtung motivationaler Merkmale die kognitive Last beim Lernen mit Multimedia beeinflusst werden kann. Manche erwarten, dass die Implementierung motivationaler Komponenten in multimedialen Lernumgebungen die kognitive Last vergrössert und daher die lernrelevante Informationsverarbeitung vermindert wird. Lernende in multimedialen Lernumgebungen, die mit motivationalen Elementen konfrontiert werden, könnten von der kognitiven Informationsverarbeitung abgelenkt werden. Negative motivationale Wirkungen können zum Beispiel dadurch verhindert werden, indem man Information in strukturierte
Kontexte einbettet, zum Entdecken anregt und indem man Information auf das Wesentliche reduziert.

Spezifische Voraussagen in Hinblick auf überfliussige Lerndetails und motivationale Adaptivität. Zwei Hauptaspekte werden für die künftige Forschung und die Lernumgebungsgestaltung in motivationaler Hinsicht besonders wichtig sein. Zum einen das Phänomen der überflüssigen Lerndetails («seductive details», Harp/Mayer, 1998) und zum anderen motivationale Adaptivität (Song/Keller, 2001). Überflüssige Lerndetails wie z. B. interessante, aber irrelevante Zusätze im multimedialen Lernmaterial lenken den Lernenden ab oder zerstören die Kohärenz des Lernprozesses. In künftigen Forschungsstudien muss abgeklärt werden, in welchem Ausmass motivationale Strategien zu diesen überflüssigen Lerndetails zählen und wie motivationale Strategien im Bereich Multimedia realisiert werden können, ohne das Risiko einzugehen, «überflüssig» zu sein, speziell in lernrelevanter Hinsicht. Es gibt dabei unterschiedliche Prognosen (vgl. Harp/Mayer, 1998): Motivationale multimediale Elemente könnten die Motivation erhöhen, aber das Lernen vermindern, indem die selektive Aufmerksamkeit der Lernenden von der relevanten Information abgelenkt wird (Unaufmerksamkeitshypothese), indem man den Übergang von einer Hauptidee zur nächsten unterbricht (Unterbrechungshypothese) oder indem man ein zusammenhängendes mentales Modell aufbaut, darin jedoch strukturell wichtige Ideen fehlen (Ablenkungshypothese).

Eine zweite wichtige Forschungsfrage sollte sich damit befassen, wie Multimedia auf die unterschiedlichen Lernenden und ihre Bedürfnisse verstärkt «adaptiv» gemacht werden kann. Eine Form der Adaptivität kann im Bereich Multimedia relativ leicht realisiert werden - indem man Fragen und Übungen auf unterschiedlichen Schwierigkeitsstufen zur Verfügung stellt (Astleitner/Keller, 1995). Allerdings ist die Aufgabenschwierigkeit auch eine wichtige Variable bei der Unterstützung des Lernens und des Wissenserwerbes. In dieser Hinsicht müssen für das Darbieten und Auswählen von Aufgaben mit unterschiedlichem Schwierigkeitsgrad Strategien gefunden werden, die einander ergänzen. Beides - die Unterstützung beim kognitiven Lernen und die Anregung von Motivation - muss berücksichtigt werden. Eine weitere offene Frage betrifft den Aspekt der Intensität von motivationalen Gestaltungen innerhalb multimedialer Umgebungen. Hier können viele unterschiedliche motivationale Taktiken realisiert werden, um ein breites Angebot von multimedialen Motivationsmöglichkeiten zur Verfügung zu stellen, sodass jeder Lernende etwas finden kann, was seinem 
Bedarf entspricht (maximale motivationale Adaptivität). Eine zu hohe Intensität an motivationalen Strategien kann zu negativen Wirkungen auf die kognitive Last und das Lernen führen. Deswegen sollte sich die Forschung auf jene multimedialen Lernumgebungen konzentrieren, die mit wenigen motivationalen Strategien ausgestattet sind (minimale motivationale Adaptivität).

Sowohl allgemeine, als auch spezifische Voraussagen stellen mehr oder minder gut fundierte Hypothesen dar, die in experimentellen oder quasi-experimentellen Untersuchungen weiter analysiert werden können.

\section{Diskussion}

Das dargestellte erweiterte Modell motivationalen multimedialen Lernens integriert eine Reihe von Forschungsergebnissen, was dem Modell mehr einen klassifikatorischen als erklärenden und integrierenden Charakter gibt. Allerdings gibt es spezifische Voraussagen aus dem Modell, die für künftige Forschungen als Hypothesen benutzt werden können.

Im erweiterten Modell zeigt der Informationsfluss keine expliziten Pfeile, was bedeutet, dass es hier noch keine eindeutigen Verbindungen gibt (vgl. Abbildung 1). Die meisten Verbindungen zwischen den postulierten Bestandteilen werden als wechselseitig angenommen. Beispielsweise nimmt man an, dass motivationale Prozesse die Aufmerksamkeit beeinflussen. Wenn es die Möglichkeit gibt, ein bestimmtes Ziel mit einer Handlung zu erreichen, dann wird die Aufmerksamkeit auf eben jene Handlung konzentriert. Weiters spielt Aufmerksamkeit aber auch eine grosse Rolle bei der Handlungskontrolle und beeinflusst motivationale Prozesse. Alle Komponenten und Verbindungen sind derzeit prinzipiell offen für weitere konzeptuelle Verfeinerungen und experimentelle Prüfungen.

Für einige Bestandteile des Modells muss auch überprüft werden, ob sie stabile und/oder instabile Persönlichkeitseigenschaften bzw. Teile der Informationsverarbeitung innerhalb des Arbeitsgedächtnisses darstellen. Aufmerksamkeit ist einerseits etwas, das informationsverarbeitende Ressourcen benötigt, andererseits verteilt die Aufmerksamkeit solche Ressourcen im Arbeitsgedächtnis durch die zentrale Exekutive. Kognitives Engagement und Monitoring sind Teile des Arbeitsgedächtnisses und stellen mehr oder weniger auch ein Persönlichkeitsmerkmal dar. Zielsetzung und Handlungskontrolle werden einerseits in informationsverarbeitende Prozesse eingebettet, die Speicherkapazität benötigen und die andererseits aber auch mit Persönlichkeitsmerkmalen verbunden sind.
Das dargestellte Modell sollte durch die Erweiterung um fünf zusätzliche Variablen (Aufmerksamkeit, Engagement, Monitoring, Zielsetzung und Handlungskontrolle) des Modells von Mayer (2001) weitere Forschungsstudien im Bereich multimedialen Lernens anregen. Ein weiteres Anliegen des erweiterten Modells liegt in der praktischen Anleitung für Mediendesigner und zwar durch die Darstellung der wichtigsten Wirkungsfaktoren für die Gestaltung von multimedialen Lernumgebungen und damit für die Optimierung des Lernens.

\section{Literatur}

Astleitner, Hermann; Keller, John. A model for motivationally adaptive computer-assisted instruction. In: Journal of Research on Computing in Education, 27 (1995): S. 270-280.

Astleitner, Hermann; Leutner, Detlev. Designing instructional technology from an emotional perspective. Journal of Research on Computing in Education, 32 (2000): S. 497-510.

Bower, Gordon. Commentary on mood and memory. In: Behaviour Research and Therapy, 25 (1987): S. 443-455.

Harp, Shannon; Mayer, Richard. The role of interest in learning from scientific text and illustrations. On the distinction between emotional interest and cognitive interest. In: Journal of Educational Psychology, 89 (1997): S. 92-101.

Hede, Andy. An integrated model of multimedia effects on learning. In: Journal of Educational Multimedia and Hypermedia, 11 (2002): S. 177191.

Hoogeveen, Martijn. Towards a theory of the effectiveness of multimedia systems. In: International Journal of Human Computer Interaction, 9 (1997): S. 151-168.

Keller, John. Motivation in cyber learning environments. In: International Journal of Educational Technology, 1 (1999): S. 7-30.

Kuhl, Julius. «Volitional mediators of cognition-behavior consistency. Self-regulatory processes and action versus state orientation.» In: Action control. From cognition to behavior. Hrsg. v. Julius Kuhl; Jürgen Beckmann. Berlin: Springer, 1985. S. 101-128.

Liao, Yuen. Effects of hypermedia on students' achievement. A metaanalysis. In: Journal of Educational Multimedia and Hypermedia, 8 (1999): S. 255-277. 
MedienPädagogik

Malone, Thomas; Lepper, Mark. «Making learning fun. A taxonomy of instrinsic motivations for learning.» In: Aptitude, learning, and instruction. Volume 3: Conative and affective process analyses. Hrsg. v. Richard Snow; Marshall Farr. Hillsdale, NJ: Erlbaum, 1987. S. 223-253.

Mayer, Richard. Multimedia learning. Cambridge: University Press, 2001.

Miles, Matthew; Huberman, Michael. Qualitative Data Analysis: An Expanded Sourcebook, Second Edition. Thousand Oaks: Sage Publications, 1994.

Plass, Jan; Chun, Dorothy; Mayer, Richard; Leutner, Detlev. Supporting visual and verbal learning preferences in a second language multimedia learning environment. In: Journal of Educational Psychology, 90 (1998): S. 25-36.

Rheinberg, Falko; Vollmeyer, Regina; Rollett, Wolfram (2000). «Motivation and action in self-regulated learning.» In: Handbook of selfregulation. Hrsg. v. Monique Boekaerts; Paul Pintrich; Moshe Zeidner. San Diego, CA: Academic Press. 2000. S. 503-529.

Song, Sang; Keller, John. Effectiveness of motivationally adaptive computer-assisted instruction on the dynamic aspects of motivation. In: Educational Technology, Research, and Development, 49 (2001): S. 522. 